\title{
A case-matched study of stereotactic radiosurgery for patients with brain metastases: comparing treatment results for those with versus without neurological symptoms
}

\author{
Takao Koiso $^{1,2} \cdot$ Masaaki Yamamoto,3 - Takuya Kawabe ${ }^{4} \cdot$ Shinya Watanabe $^{1,5}$. \\ Yasunori Sato $^{6} \cdot$ Yoshinori Higuchi $^{7}$ - Tetsuya Yamamoto ${ }^{2} \cdot$ Akira Matsumura $^{2}$. \\ Hidetoshi Kasuya $^{3} \cdot$ Bierta E. Barfod $^{1}$
}

Received: 20 April 2016 / Accepted: 27 August 2016 / Published online: 3 September 2016

(C) The Author(s) 2016. This article is published with open access at Springerlink.com

\begin{abstract}
We aimed to reappraise whether post-stereotactic radiosurgery (SRS) results for brain metastases differ between patients with and without neurological symptoms. This was an institutional review board-approved, retrospective cohort study using our prospectively accumulated database including 2825 consecutive BM patients undergoing gamma knife SRS alone during the 15-year period since July 1998. The 2825 patients were divided into two groups; neurologically asymptomatic [group A, 1374 patients $(48.6 \%)]$ and neurologically symptomatic [group B, 1451 (51.4\%)]. Because there was considerable
\end{abstract}

Masaaki Yamamoto

bcd06275@nifty.com

1 Katsuta Hospital Mito GammaHouse, 5125-2 Nakane, Hitachi-naka, Ibaraki 312-0011, Japan

2 Department of Neurosurgery, Faculty of Medicine, University of Tsukuba, 2-1-1 Amakubo, Tsukuba, Ibaraki 305-8576, Japan

3 Department of Neurosurgery, Tokyo Women's Medical University Medical Center East, 8-1-10 Nishiogu, Arakawaku, Tokyo 104-0045, Japan

4 Department of Neurosurgery, Kyoto Prefectural University of Medicine, Graduate School of Medical Sciences, 465 Kawaramachi-Hirokoji, Kamigyo-ku, Kyoto 602-8566, Japan

5 Department of Neurosurgery, National Hospital Organization Mito Medical Center, 280 Sakuranosato, Ibaraki-machi, Ibaraki 311-3193, Japan

6 Clinical Research Center, Chiba University Graduate School of Medicine, 1-8-1 Inohana, Chuo-ku,

Chiba 260-8677, Japan

7 Department of Neurological Surgery, Chiba University Graduate School of Medicine, 1-8-1 Inohana, Chuo-ku, Chiba 260-8677, Japan bias in pre-SRS clinical factors between groups A and B, a case-matched study was conducted. Ultimately, 1644 patients (822 in each group) were selected. The standard Kaplan-Meier method was used to determine post-SRS survival. Competing risk analysis was applied to estimate cumulative incidences of neurological death, neurological deterioration, local recurrence, re-SRS for new lesions and SRS-induced complications. Post-SRS median survival times (MSTs) did not differ between the two groups; 7.8 months in group A versus 7.4 months in group B patients (HR 1.064, 95\% CI 0.963-1.177, $\mathrm{p}=0.22$ ). However, cumulative incidences of neurological death (HR 1.637, 95\% CI 1.174-2.281, p=0.0036) and neurological deterioration (HR 1.425, 95\% CI 1.073-1.894, $\mathrm{p}=0.014$ ) were significantly lower in the group $\mathrm{A}$ than in the group $\mathrm{B}$ patients. Neurologically asymptomatic patients undergoing SRS for BM had better results than symptomatic patients in terms of both maintenance of good neurological state and prolonged neurological survival. Thus, we conclude that screening computed tomography/magnetic resonance imaging is highly beneficial for managing cancer patients.

Keywords Brain metastases - Radiation therapy · Radiosurgery $\cdot$ Gamma knife $\cdot$ Metastatic tumor

\section{Introduction}

Brain metastases (BMs) are generally a life-threatening neurological problem for cancer patients regardless of primary tumor sites [1-4]. However, due mainly to recent advances in systemic cancer treatment $[5,6]$, an appropriately selected BM patient subgroup can now achieve longer survival with maintenance of good neurological function if BMs are well controlled. It is now widely recognized 
that not only prolonged survival but also maintenance of good neurological function is crucial for managing patients with BMs. Thus, successful management requires precise BM diagnosis in an early disease stage, i.e., before a BM manifests with neurological symptoms. Figlin et al. reported BM to be discovered within 2 years after initial treatment of lung cancer [7]. Moreover, with widespread use of computed tomography (CT) and/or magnetic resonance images (MRI), increasing numbers of patients with asymptomatic BMs are being diagnosed [5, 6, 8-10]. Most notably, MRI is more sensitive than $\mathrm{CT}$ for $\mathrm{BM}$ detection $[5,10-14]$. BMs were reportedly found in 6-30\% of initially-diagnosed lung cancer patients $[6,10,15-19]$ and were neurologically silent in $14 \%$ [5].

As Lippitz et al. recently reviewed extensively, stereotactic radiosurgery (SRS) has become an established treatment option for managing BM patients [20]. SRS is more advantageous than other treatments, i.e., whole brain radiotherapy (WBRT), surgery, systemic anti-cancer agent therapy and combinations of these modalities, in terms of costs, hospitalization, morbidity, mortality and wider applicability and repeatability [21]. With all cancer treatment modalities, it is generally believed that both early diagnosis and immediate treatment improve patient outcomes. Although considerable numbers of series, both prospective and retrospective, have demonstrated treatment results including neurological survival and maintenance of neurological condition in BM patients undergoing SRS, little is known about whether post-SRS results for BM differ between patients with and without neurological symptoms. We aimed to reappraise whether neurologically-asymptomatic patients are more likely to benefit from SRS, in terms of both neurological survival and maintenance of neurological condition, than symptomatic patients.

\section{Materials and methods}

\section{Patient population}

This was an institutional review board (IRB)-approved, retrospective cohort study using our prospectively accumulated database including 2825 consecutive BM patients undergoing gamma knife SRS alone during the 15-year period between July 1998 and June 2013 (Tokyo Women's Medical University IRB: \#1981). As all patients had been referred to us for SRS, their primary physicians had mostly made the patient selections. Patient selection criteria may thus have differed among referring physicians. Therefore, one author (MY) decided whether or not to accept a patient. We did not perform SRS on patients with low Karnofsky Performance Status (KPS) scores $(<70 \%)$ due to systemic diseases, a non-cooperative state due to poor neurocognitive function, meningeal dissemination, or an anticipated survival period of 3 months or less. As to tumor size, if MRI demonstrated tumors with diameters of $2-3 \mathrm{~mm}$ in the brainstem or the optic apparatus, we performed SRS rather than further follow-up observation. Otherwise, SRS was usually postponed with close MRI follow-up until the tumor diameter exceeded approximately $1 \mathrm{~cm}$. Table 1 summarizes clinical characteristics, overall and for the two groups, i.e. neurologically asymptomatic (group A, 1374 patients) and symptomatic (group B, 1451 patients). As mentioned above, the primary physicians responsible for each patient decided the indications for both surgery and radiotherapy. Therefore, prior to SRS, among the 2825 patients, $523(18.5 \%)$ had undergone surgical removal of brain METs and 141 (4.8\%) WBRT (Table 1).

Before SRS, the treatment strategies were explained in detail to each patient, as well as at least one adult relative, and written informed consent was obtained from all patients before SRS by the second author (M.Y.). As our previous report described our radiosurgical techniques in detail, they are not repeated herein $[22,23]$. Briefly, standard SRS procedures were performed using a Leksell gamma unit Model B before June, 2003, and thereafter a Leksell gamma unit Model C (Elekta AB, Stockholm, Sweden).

Post-SRS, all patients were routinely managed by referring physicians and were recommended to have clinical and neuro-imaging examinations at an approximately $2-3$ month interval. Neuroimaging follow-up could not be performed in 815 patients $(28.9 \%)$; mostly because of post-SRS early deterioration due to systemic diseases [median survival time of this subset was 2.5 (95\% CI 2.3-2.6 months)]. Approximately $50 \%$ of our 2825 patients came to our outpatient clinic periodically, while clinical and/or neuroimaging data were sent to us by post in about $25 \%$ of cases. The second author (M.Y.) called the remaining $25 \%$ of patients or their relatives by telephone to confirm the patients' conditions. For deceased patients, the day of death, cause of death, and detailed information on patient condition changes were requested by telephone.

\section{Clinical outcomes}

As clinical outcomes were described in detail in our previous report, they are not repeated herein [22, 23]. Briefly, the primary endpoint was overall survival, and the secondary endpoints were neurological death, neurological deterioration, local recurrence of the treated tumor, repeat SRS for new lesions, salvage WBRT, salvage surgery, and SRSinduced major complications. For each endpoint, failures were regarded as events and any others as censored. Overall survival time was defined as the interval between the first SRS and death due to any cause (progression of systemic metastases and/or BM, other disease unrelated to cancer, 
Table 1 Summary of clinical characteristics of 2825 brain metastasis patients (cohort)

\begin{tabular}{|c|c|c|c|c|}
\hline \multirow[t]{2}{*}{ Characteristics } & \multirow[t]{2}{*}{ Total } & \multicolumn{2}{|c|}{ Neurological symptoms } & \multirow{2}{*}{$\begin{array}{l}\mathrm{p} \\
\text { values }^{\mathrm{a}}\end{array}$} \\
\hline & & No (group A) & Yes (group B) & \\
\hline No. of patients & 2825 & 1374 & 1451 & \\
\hline \multicolumn{5}{|l|}{ Age (years) } \\
\hline Mean & 64 & 64 & 65 & 0.05 \\
\hline Range & $19-96$ & $19-96$ & $19-93$ & \\
\hline \multicolumn{5}{|l|}{ Sex } \\
\hline Female & $1134(40.1 \%)$ & $547(39.8 \%)$ & $587(40.5 \%)$ & 0.73 \\
\hline \multicolumn{5}{|l|}{ Tumor numbers } \\
\hline Mean & 7 & 7 & 7 & 0.45 \\
\hline Median & 3 & 3 & 3 & \\
\hline Range & $1-89$ & $1-69$ & $1-89$ & \\
\hline IQR & $1-8$ & $1-8$ & $1-8$ & \\
\hline \multicolumn{5}{|c|}{ Primary cancer sites } \\
\hline Lung & $1840(65.1 \%)$ & $1046(76.1 \%)$ & $794(54.7 \%)$ & $<0.001^{\mathrm{b}}$ \\
\hline Breast & $309(10.9 \%)$ & $122(8.9 \%)$ & $187(12.9 \%)$ & \\
\hline GI tract & $328(11.6 \%)$ & $87(6.3 \%)$ & $241(6.9 \%)$ & \\
\hline Kidney & $115(4.1 \%)$ & $47(3.4 \%)$ & $68(3.3 \%)$ & \\
\hline Others & $233(5.7 \%)$ & $72(5.2 \%)$ & $161(11.1 \%)$ & \\
\hline \multicolumn{5}{|c|}{ Primary cancer status } \\
\hline Controlled & $865(30.7 \%)$ & $414(30.1 \%)$ & $451(31.1 \%)$ & 0.60 \\
\hline \multicolumn{5}{|c|}{ Extra-cerebral METs } \\
\hline No & $1456(51.5 \%)$ & $695(50.6 \%)$ & $679(49.4 \%)$ & 0.33 \\
\hline \multicolumn{5}{|l|}{ KPS } \\
\hline$\geq 80 \%$ & $2150(76.1 \%)$ & $1176(85.6 \%)$ & $974(67.1 \%)$ & $<0.001$ \\
\hline \multicolumn{5}{|c|}{ Modified-RPA class $[34,35]$} \\
\hline $1+2 \mathrm{a}$ & $638(22.6 \%)$ & $363(26.4 \%)$ & $275(19.0 \%)$ & \\
\hline $2 b$ & $872(30.9 \%)$ & $457(33.3 \%)$ & $415(28.6 \%)$ & $0.08^{\mathrm{c}}$ \\
\hline $2 c+3$ & $1315(46.5 \%)$ & $554(40.3 \%)$ & $761(52.5 \%)$ & $<0.001^{\mathrm{C}}$ \\
\hline \multicolumn{5}{|l|}{ Prior surgery } \\
\hline Yes & $523(18.5 \%)$ & $113(8.2 \%)$ & $410(28.3 \%)$ & $<0.001$ \\
\hline \multicolumn{5}{|l|}{ Prior WBRT } \\
\hline Yes & $141(5.0 \%)$ & $85(6.2 \%)$ & $56(3.9 \%)$ & 0.006 \\
\hline \multicolumn{5}{|c|}{ Tumor volume (cc) } \\
\hline \multicolumn{5}{|l|}{ Cumulative } \\
\hline Mean & 9.74 & 6.43 & 12.87 & $<0.001$ \\
\hline Range & $0.01-126.2$ & $0.01-115.3$ & $0.04-126.2$ & \\
\hline IQR & $1.89-12.92$ & $0.89-8.21$ & $3.69-17.45$ & \\
\hline \multicolumn{5}{|l|}{ Largest tumor } \\
\hline Mean & 6.78 & 4.42 & 9.02 & $<0.001$ \\
\hline Range & $0.01-94.20$ & $0.01-94.20$ & $0.02-89.30$ & \\
\hline IQR & $1.10-8.81$ & $0.52-5.22$ & $2.60-11.80$ & \\
\hline \multicolumn{5}{|c|}{ Peripheral dose (Gy) } \\
\hline Mean & 21.12 & 22.01 & 20.52 & $<0.001$ \\
\hline Range & $10.00-32.00$ & $12.00-32.00$ & $10.00-25.00$ & \\
\hline IQR & $20.00-24.00$ & $20.00-24.00$ & $18.00-24.00$ & \\
\hline
\end{tabular}

IQR interquartile range, GI gastrointestinal, METs metastases, KPS Karnofsky Performance Status, ${ }^{12} R P A$ recursive partitioning analysis, ${ }^{4} W B R T$ whole brain radiotherapy, $C I$ confidence interval

${ }^{a}$ Student $t$ test was used for continuous variables and Fisher's exact test for pairs of categorical variables, ${ }^{\mathrm{b}}$ Lung versus non-lung, ${ }^{\mathrm{c}}$ Modified-RPA classes $1+2 \mathrm{a}$ versus $2 \mathrm{~b},{ }^{\mathrm{d}}$ Modified-RPA classes $2 \mathrm{~b}$ versus $2 \mathrm{c}+3$ 
accident, suicide, and so on) or the day of the last follow-up. Neurological death was defined as death caused by any intracranial disease, including tumor recurrence, carcinomatous meningitis, cerebral dissemination, and progression of other untreated intracranial tumors. Neurological deteriorationfree survival time was defined as the interval between the first SRS and the day that any brain disease-caused neurological worsening manifested (that is, local recurrence, progression of new lesions, and SRS-induced complications). Decreases in KPS scores, in patients with scores of $20 \%$ or less, due to neurological worsening were regarded as events and any others as censored. Major complication-free survival time was taken as the interval between the first SRS and the day major SRS-induced complications occurred. Patients with major complications included those with Radiation Therapy Oncology Group (RTOG) neurotoxicity grades of 2 or worse and, even if the grade was either 0 or 1 , those in whom surgical intervention was required based on sequential MRI follow-up demonstrating progressive enlargement of a cyst and/or a mass lesion with further observation thus being regarded as excessively high risk; all of these conditions were regarded as events and any others as censored [24].

\section{Statistical analysis}

All data were analyzed according to the intention-to-treat principle. For baseline variables, summary statistics were constructed using frequencies and proportions for categorical data and medians, ranges and interquartile ranges (IQR) for continuous variables. We compared patient characteristics using the Fisher exact test for categorical outcomes and t-tests for continuous variables, as appropriate. The KaplanMeier method was used to estimate overall survival. Moreover, univariate analysis using the Cox proportional hazard model was performed to determine pre-SRS clinical factors favoring longer survival.

For time-to-event outcomes, the cumulative incidences of neurological death, neurological deterioration, local recurrence, repeat SRS, and major complications were estimated by a competing risk analysis, because death is a competing risk for loss to follow-up (that is, patients who die can no longer become lost to follow-up) [25-27]. Also, to identify baseline and clinical variables associated with the 5 aforementioned outcomes, competing risk analyses were performed with the Fine-Gray generalization of the proportional hazards model accounting for death as a competing risk [28]. Fine-Gray generalization makes use of the subdistribution hazard to model cumulative incidence, thereby quantifying the overall benefit or harm of an exposure [29].

All comparisons were planned and the tests were twosided. A $p$ value of $<0.05$ was considered to be statistically significant. All statistical analyses were performed by a statistician (YS) using SAS software version 9.4 (SAS Institute, Cary, NC, USA) and the R statistical program, version 3.10. Before statistical analyses, the database was cleaned (by YH). These two authors were not involved in either SRS treatment or patient follow-up.

\section{Results}

\section{Cohort studies}

Four patients were lost to follow-up $(0.34 \%$, three in group A and one in group B). As of January 2014, the median post-SRS observation time among censored observations (212 patients) was 41.9 (IQR 8.7-54.0, maximum 173.5) months and 2609 patients $(92.4 \%)$ had died. The overall median survival time (MST) after SRS was 7.6 months (95\% CI 7.3-8.0 months). Actuarial post-SRS survival rates
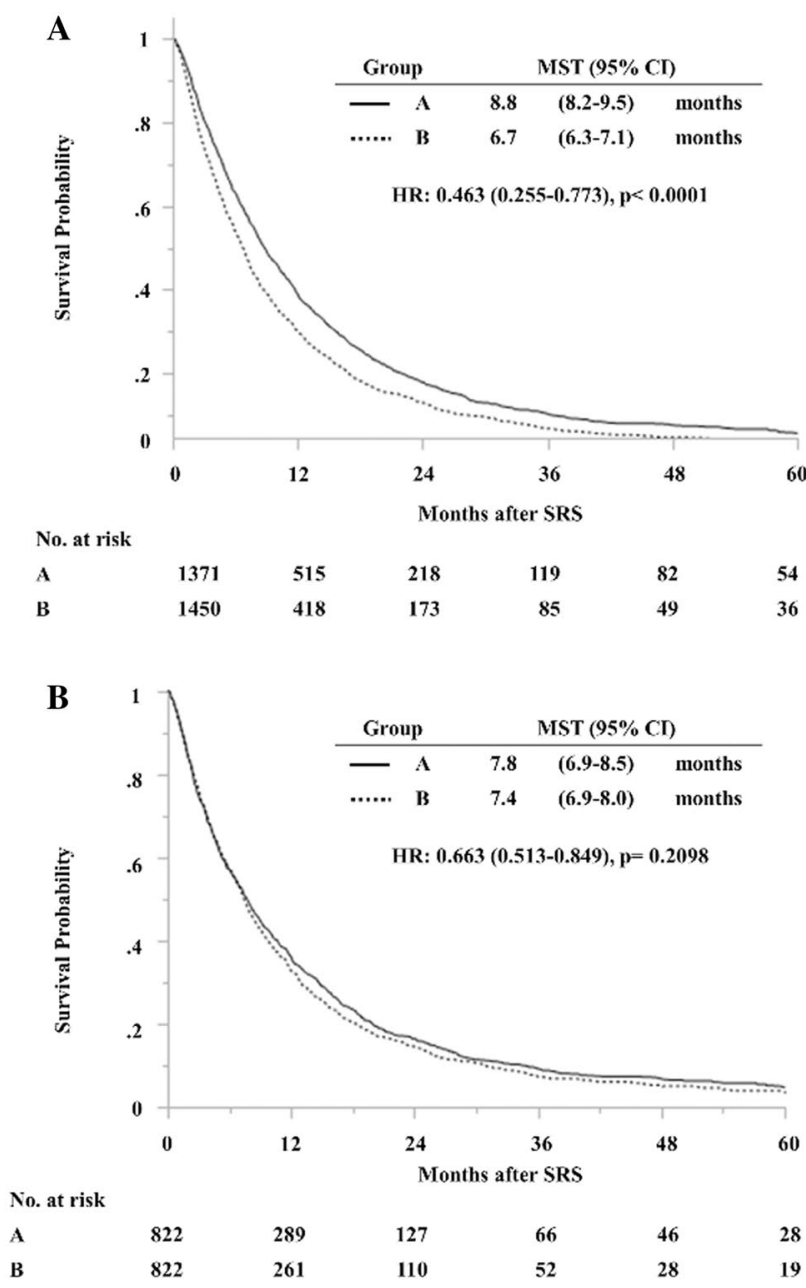

Fig. 1 Overall survival based on 2825 patients (a) and on a subset of 1644 case-matched patients (b) according to neurological symptoms [asymptomatic (group A) and symptomatic (group B)], estimated using the standard Kaplan-Meier method 
Table 2 Summary of clinical characteristics of 1644 case-matched brain metastasis patients

\begin{tabular}{|c|c|c|c|c|}
\hline \multirow[t]{2}{*}{ Characteristics } & \multirow[t]{2}{*}{ Total } & \multicolumn{2}{|c|}{ Neurological symptoms } & \multirow{2}{*}{$\begin{array}{l}\mathrm{p} \\
\text { values }\end{array}$} \\
\hline & & No (group A) & Yes (group B) & \\
\hline No. of patients & 1644 & 822 & 822 & \\
\hline \multicolumn{5}{|l|}{ Age (years) } \\
\hline Mean & 64 & 64 & 65 & 0.04 \\
\hline Range & $19-96$ & $19-96$ & $19-93$ & \\
\hline \multicolumn{5}{|l|}{ Sex } \\
\hline Female & $650(39.5 \%)$ & $327(39.8 \%)$ & $323(39.3 \%)$ & 0.88 \\
\hline \multicolumn{5}{|l|}{ Tumor numbers } \\
\hline Mean & 7 & 8 & 7 & 0.87 \\
\hline Median & 3 & 4 & 3 & \\
\hline Range & $1-89$ & $1-63$ & $1-74$ & \\
\hline IQR & $1-9$ & $1-9$ & $1-8$ & \\
\hline \multicolumn{5}{|c|}{ Primary cancer sites } \\
\hline Lung & $1077(65.5 \%)$ & $531(64.6 \%)$ & $546(66.4 \%)$ & $0.47^{\mathrm{a}}$ \\
\hline Breast & $192(11.7 \%)$ & $101(12.3 \%)$ & $91(11.1 \%)$ & \\
\hline GI tract & $172(10.5 \%)$ & $87(10.6 \%)$ & $85(10.3 \%)$ & \\
\hline Kidney & $79(4.8 \%)$ & $42(5.1 \%)$ & $37(4.5 \%)$ & \\
\hline Others & $124(7.5 \%)$ & $61(7.4 \%)$ & $63(7.7 \%)$ & \\
\hline \multicolumn{5}{|c|}{ Primary cancer status } \\
\hline Controlled & $507(30.8 \%)$ & $254(30.9 \%)$ & $253(30.8 \%)$ & 1.00 \\
\hline \multicolumn{5}{|c|}{ Extra-cerebral METs } \\
\hline No & $830(50.5 \%)$ & $412(50.1 \%)$ & $418(50.9 \%)$ & 0.81 \\
\hline \multicolumn{5}{|l|}{ KPS } \\
\hline$\geq 80 \%$ & $1291(78.5 \%)$ & $647(78.7 \%)$ & $644(78.4 \%)$ & 0.90 \\
\hline \multicolumn{5}{|c|}{ Modified-RPA class $[34,35]$} \\
\hline $1+2 a$ & $354(21.5 \%)$ & $172(20.9 \%)$ & $182(22.1 \%)$ & \\
\hline $2 b$ & $528(32.1 \%)$ & $272(33.1 \%)$ & $256(31.1 \%)$ & $0.41^{\mathrm{c}}$ \\
\hline $2 c+3$ & $762(46.4 \%)$ & $378(46.0 \%)$ & $384(46.7 \%)$ & $0.53^{\mathrm{d}}$ \\
\hline \multicolumn{5}{|l|}{ Prior surgery } \\
\hline Yes & $231(14.1 \%)$ & $113(13.8 \%)$ & $118(14.4 \%)$ & 0.78 \\
\hline \multicolumn{5}{|l|}{ Prior WBRT } \\
\hline Yes & $75(4.6 \%)$ & $36(4.4 \%)$ & $39(4.7 \%)$ & 0.81 \\
\hline \multicolumn{5}{|c|}{ Tumor volume (cc) } \\
\hline \multicolumn{5}{|l|}{ Cumulative } \\
\hline Mean & 8.92 & 8.80 & 9.04 & 0.87 \\
\hline Range & $0.01-115.3$ & $0.01-115.3$ & $0.05-77.88$ & \\
\hline IQR & $2.18-11.74$ & $1.80-11.66$ & $2.62-11.95$ & \\
\hline \multicolumn{5}{|l|}{ Largest tumor } \\
\hline Mean & 6.17 & 6.06 & 6.29 & 0.65 \\
\hline Range & $0.02-94.20$ & $0.02-94.20$ & $0.05-70.00$ & \\
\hline IQR & $1.31-7.80$ & $0.92-7.82$ & $1.80-7.80$ & \\
\hline \multicolumn{5}{|c|}{ Peripheral dose (Gy) } \\
\hline Mean & 21.32 & 22.28 & 21.30 & 0.55 \\
\hline Range & $10.00-25.00$ & $12.00-32.00$ & $12.00-25.00$ & \\
\hline IQR & $20.00-24.00$ & $20.00-24.00$ & $20.00-24.00$ & \\
\hline
\end{tabular}

IQR interquartile range, GI gastrointestinal, METs metastases, KPS Karnofsky Performance Status ${ }^{12} R P A$ recursive partitioning analysis, ${ }^{4} W B R T$ whole brain radiotherapy, $C I$ confidence interval

${ }^{a}$ Student $t$ test was used for continuous variables and Fisher's exact test for pairs of categorical variables, ${ }^{b}$ Lung versus non-lung, ${ }^{\mathrm{c}}$ Modified-RPA classes $1+2 \mathrm{a}$ versus $2 \mathrm{~b},{ }^{\mathrm{d}}$ Modified-RPA classes $2 \mathrm{~b}$ versus $2 \mathrm{c}+3$ 
Table 3 Summary of treatment results after stereotactic radiosurgery (SRS)

\begin{tabular}{llll}
\hline & \multicolumn{2}{l}{ Neurological symptoms } & $\mathrm{p}$ \\
\cline { 2 - 3 } & No (group A) & Yes (group B) & \\
\hline No. of patients & 822 & 822 & \\
Neurological death $^{\mathrm{a}}$ & $58(7.9 \%)$ & $88(11.9 \%)$ & 0.01 \\
Neurological deterioration & $84(10.2 \%)$ & $110(13.4 \%)$ & 0.06 \\
Local recurrence $^{\mathrm{b}}$ & $48(8.5 \%)$ & $57(9.5 \%)$ & 0.54 \\
Repeat SRS & $259(31.5 \%)$ & $224(27.3 \%)$ & 0.07 \\
Salvage WBRT & $36(4.4 \%)$ & $23(2.8 \%)$ & 0.11 \\
Salvage surgery & $14(1.7 \%)$ & $15(1.8 \%)$ & 1.00 \\
SRS-related complications & $30(3.7 \%)$ & $24(2.9 \%)$ & 0.49 \\
\hline
\end{tabular}

${ }^{a}$ Based on 1471 [734 (96.0\%) in group A and 737 (96.6\%) in group B, $\mathrm{p}=0.59$ ] deceased patients whose causes of death were determined (57 patients were excluded because causes of death were not available)

${ }^{b}$ Based on 1165 [567 (69.0\%) in group A and 598 (72.8\%) in group B, $\mathrm{p}=0.10]$ patients ( 479 patients were excluded because neuro-imaging results were not available)

were $58.6,34.3,15.4,8.6$, and $4.7 \%$ at the 6 th, 12 th, 24 th, 36 th, and 60 th post-SRS month, respectively. Among the 2609 deceased patients, causes of death could not be determined in 109 , but were confirmed in the remaining 2500 to be non-brain disease in $2211(88.4 \%)$ and brain disease in $289(11.6 \%)$. MST after SRS was significantly longer in the 1374 group A (8.9 months) than in the 1451 group B (6.7 months) patients (HR 1.238, 95\% CI 1.146-1.337, $\mathrm{p}<0.001$ ) (Fig. 1).

Among 1840 patients with lung cancer, BMs had not manifested with neurological symptoms at the time of SRS in 1046 (56.9\%). However, 39.5\% (122/309 patients) of breast cancer, $26.5 \%(87 / 328)$ of gastro-intestinal tract cancer and $40.9 \%(47 / 115)$ of renal cancer patients underwent SRS for asymptomatic BMs.

\section{Studies of case-matched subset}

As shown in Table 1, proportions of patients with some of the clinical characteristics differed significantly between groups A and B, i.e., proportions of primary cancer sites, KPS, Modified-RPA classes, and prior treatments (surgery and WBRT) were strikingly uneven and both cumulative tumor volumes and the volumes of the largest tumors differed significantly between the two groups. These differences might have impacted survival and/or neurological deterioration. Therefore, a case-matched study was conducted by one of the authors (Y.S.), who did not participate in other aspects of this study and was blinded to final outcomes. Patient selection was performed employing the propensity score matching method with a Greedy 5-To-1 Digit-Matching algorithm [30] for clinical factors (primary tumor sites, Karnofsky score, Modified-Recursive Partitioning Analysis classes, prior procedures [surgery and WBRT], cumulative tumor volume, volume of the largest tumor, and peripheral doses), all of which had $p$ values $<0.05[31$, 32]. After all of the propensity-score matches had been performed, we compared baseline characteristics between the two groups. Ultimately, 1644 patients (822 in each group) were selected. The $p$ values after matching were over 0.05 for all clinical factors except age (Table 2). However, the difference in mean ages was only 1.2 years (63.8 years/ group A vs. 65.0 years/group B). This difference is not considered to be clinically meaningful.

As shown in Fig. 1, although the post-SRS MST was slightly longer in group A (7.8 months) than in group B (7.4 months) patients, this difference was not statistically significant (HR 1.064, 95\% CI 0.963-1.177, $\mathrm{p}=0.22$ ). Both crude and cumulative incidences of neurological death were significantly lower in group A than in the group B patients (HR 1.637, 95\% CI 1.174-2.281, p=0.0036) (Tables 3, 4). Although crude incidences of neurological deterioration did not differ significantly between the two patient groups, cumulative incidences of neurological deterioration were significantly lower in the group A than in the group B patients (HR 1.425, 95\% CI 1.073-1.894, $\mathrm{p}=0.014)$.

Crude and cumulative incidences of local recurrence, repeat-SRS and SRS-related complications did not differ significantly between the two patient groups, as shown in Tables 3 and 4. Also, the crude incidence of salvage treatment, i.e., surgery or WBRT, did not differ significantly between the two patient groups (Table 3).

\section{Four major original cancers}

There were no significant MST differences between the two groups, A and B, in patients with lung, gastro-intestinal and renal cancers (Table 3). However, in those with breast cancer, the MST of the group A patients $[12.3(95 \% \mathrm{CI}$ 8.7-16.0) months] was significantly longer than that of the group $B$ patients $[8.3(6.4-10.8)$ months, $p=0.01]$. In lung cancer patients, the crude incidence of neurological death was significantly higher in the group B patients $(12.8 \%)$ than in the group A patients $(8.1 \%, p=0.02)$. However, there were no significant differences in the incidence of neurological death between the A and B groups for patients with breast, gastro-intestinal and renal cancers. Also, in lung cancer patients, the crude incidence of neurological deterioration in the group B patients $(15.2 \%)$ was significantly higher than that in the group A patients $(10.0 \%$, $\mathrm{p}=0.01$ ). There were, however, no significant differences in the incidence of neurological deterioration between groups A and B for patients with breast, gastro-intestinal and renal cancers. 
Table 4 Summary of time-toevent outcome studies using competing risk analyses

\begin{tabular}{|c|c|c|c|c|c|c|}
\hline & \multicolumn{4}{|c|}{ Cumulative incidences (post-SRS months) } & \multirow[t]{2}{*}{$\operatorname{HR}(95 \% \mathrm{CI})$} & \multirow{2}{*}{$\begin{array}{l}\mathrm{p} \\
\text { value }\end{array}$} \\
\hline & 6 & 12 & 24 & 36 & & \\
\hline Neurological death & & & & & $1.637(1.174-2.281)$ & 0.0036 \\
\hline $\begin{array}{l}\text { Asymptomatic (group } \\
\text { A) }\end{array}$ & 0.021 & 0.033 & 0.057 & 0.068 & & \\
\hline $\begin{array}{l}\text { Symptomatic (group } \\
\text { B) }\end{array}$ & 0.030 & 0.073 & 0.100 & 0.110 & & \\
\hline $\begin{array}{l}\text { Neurological } \\
\text { deterioration }\end{array}$ & & & & & $1.425(1.073-1.894)$ & 0.014 \\
\hline $\begin{array}{l}\text { Asymptomatic (group } \\
\text { A) }\end{array}$ & 0.032 & 0.057 & 0.080 & 0.096 & & \\
\hline $\begin{array}{l}\text { Symptomatic (group } \\
\text { B) }\end{array}$ & 0.047 & 0.093 & 0.121 & 0.130 & & \\
\hline Local recurrence $^{\mathrm{a}}$ & & & & & $1.360(0.926-1.998)$ & 0.11 \\
\hline $\begin{array}{l}\text { Asymptomatic (group } \\
\text { A) }\end{array}$ & 0.017 & 0.046 & 0.062 & 0.072 & & \\
\hline $\begin{array}{l}\text { Symptomatic (group } \\
\text { B) }\end{array}$ & 0.023 & 0.057 & 0.091 & 0.099 & & \\
\hline Repeat SRS & & & & & $0.880(0.736-1.053)$ & 0.16 \\
\hline $\begin{array}{l}\text { Asymptomatic (group } \\
\text { A) }\end{array}$ & 0.153 & 0.256 & 0.301 & 0.318 & & \\
\hline $\begin{array}{l}\text { Symptomatic (group } \\
\text { B) }\end{array}$ & 0.131 & 0.229 & 0.269 & 0.275 & & \\
\hline $\begin{array}{l}\text { SRS-related } \\
\text { complications }\end{array}$ & & & & & $0.088(0.514-1.507)$ & 0.64 \\
\hline $\begin{array}{l}\text { Asymptomatic (group } \\
\text { A) }\end{array}$ & 0.006 & 0.013 & 0.021 & 0.026 & & \\
\hline $\begin{array}{l}\text { Symptomatic (group } \\
\text { B) }\end{array}$ & 0.012 & 0.021 & 0.027 & 0.027 & & \\
\hline
\end{tabular}

SRS stereotactic radiosurgery, $H R$ hazard ratio, $C I$ confidence interval

${ }^{\mathrm{a}}$ Based on 1165 [567 (69.0\%) in group A and 598 (72.8\%) in group B, p=0.10] patients (479 patients were excluded because neuro-imaging results were not available)

\section{Discussion}

Debate continues as to whether post-treatment survival periods are significantly longer in neurologically asymptomatic patients than in symptomatic patients. Neurologically asymptomatic patients reportedly have better outcomes $[2$, 33-36], though some authors have argued against such a relationship [6, 17, 37]. However, all previously reported series were based on relatively small sample sizes. Furthermore, only overall survivals were discussed in prior reports, with neithor neurological survival nor maintenance of neurological condition being described. Maintenance of good neurological function and, eventually, a decreased incidence of neurological death, have recently been recognized as being crucial for managing patients with BMs. We consider our herein-reported dataset, with a relatively large sample size, to show that SRS, prior to BMs becoming neurologically symptomatic, has the potential to achieve prolonged maintenance of neurological function and to minimize neurological death, although MST did not differ significantly between the two groups.
In general, earlier diagnosis and non-delayed treatment are believed to improve treatment outcomes for patients with all types of malignant tumors. However, based on 181 patients, Seute et al. reported chemotherapies to be ineffective for asymptomatic BM from small cell lung cancer [17]. Kim et al. also reported that there was no significant difference in overall survivals between 7 asymptomatic and 31 symptomatic patients with BM from non small cell lung cancer (NSCLC) (43 vs. 45 weeks, $p=0.3689$ ) [6]. Kuba et al. obtained similar results in 53 asymptomatic and 12 symptomatic patients with BM from breast cancer (12 vs. 13 months, $p=0.99$ ) [37]. Brain CT for lung cancer patients is recommended only for those who are neurologically symptomatic, because screening neuro-imaging examinations are not cost-effective for asymptomatic patients [38, 39]. We disagree with this view. We advocate considering that once a cancer patient becomes neurologically-handicapped, medical costs far exceed that for a single neuro-imaging examination using either CT or MRI, though it must be noted that these costs vary markedly among countries, i.e., approximately US\$300 in Japan, even if a contrast study is added, 
Table 5 Number of patients with synchronous versus metachronous presentation and interval between diagnosis of primary cancer and stereotactic radiosurgery (SRS) (cohort)

\begin{tabular}{|c|c|c|c|c|c|}
\hline \multirow[t]{2}{*}{ Primary cancer sites } & \multirow[t]{2}{*}{ No. of patients } & \multicolumn{2}{|l|}{ Presentation } & \multicolumn{2}{|c|}{$\begin{array}{l}\text { Interval (months) between diagnosis of } \\
\text { primary cancer and SRS }\end{array}$} \\
\hline & & Synchronous & Metachronous & Mean/median & Maximum/IQR \\
\hline Lung & 1840 & $378(20.5 \%)$ & $1462(79.5 \%)$ & $17 / 10$ & $262 / 3-21$ \\
\hline Breast & 309 & $4(1.3 \%)$ & $305(98.7 \%)$ & $59 / 48$ & 289/27-78 \\
\hline GI tract & 328 & $25(7.6 \%)$ & $303(92.4 \%)$ & $34 / 24$ & $237 / 11-45$ \\
\hline Kidney & 115 & $10(8.7 \%)$ & $105(91.3 \%)$ & $55 / 29$ & $324 / 8-90$ \\
\hline
\end{tabular}

GI gastro-intestinal

$\mathrm{p}<0.0001$

and US\$3000 in the USA. Moreover, expenses are not the only consideration. The difficulty and anxiety experienced by neurologically-handicapped patients must also be taken into account. In fact, most physicians in Japan are now performing screening MRI for lung cancer patients, even those who are neurologically asymptomatic, as described above. We advocate that this policy be generally adopted by physicians who manage cancer patients, regardless of the original tumor type.

However, it should be kept in mind that BMs occur at a relatively early disease stage in lung cancer patients as compared to those with breast, gastro-intestinal tract or kidney cancers, as shown in Table 5 (on line only). This issue makes it difficult to perform screening MR imaging examinations for all patients with non-lung cancers. In fact, the high costs of neuro-imaging examinations are considered to be a major hurdle to routinely using CT/MRI for cancer patients without neurological symptoms in the USA. Nevertheless, as described above, SRS for non-symptomatic BMs was clearly shown to have survival benefits in breast cancer patients. As with lung cancer patients, we recommend screening neuro-imaging examinations for breast cancer patients at high risk for BMs, i.e., as reported by BarnholtzSloan et al. and Shouteen et al. who found incidences of BMs to be higher in patients with advanced disease [40, 41]. In this study, we found that performing SRS for nonsymptomatic BMs in patients with gastro-intestinal and renal cancers provided no benefits in terms of neurological death or maintenance of neurological condition. For these patients, periodic CT examinations for re-staging consistently including the brain are worthwhile for detecting BM before they manifest with neurological symptoms.

In contrast, Sanchez et al. found neurologically asymptomatic NSCLC patients to have longer survival; the MST of 12 asymptomatic patients was 7.5 months and that of 69 symptomatic patients was 4.0 months $(p=0.02)$ [34]. Moreover, they also reported that control of the neurological status of asymptomatic patients is better than that of symptomatic patients (80 vs. 40\%) [34]. However, these reports described a small number of patients. Yamamoto, Serizawa, and colleagues reported that, based on 1196 prospectively enrolled patients undergoing gamma knife SRS, there were significant MST differences in Mets between asymptomatic and symptomatic patient groups (HR 1.779, 95\% CI 1.541-1.589, $\mathrm{p}<0.0001$ ) [35]. However, in both reports, there were considerable biases between the asymptomatic and symptomatic patient groups, i.e., the tumor volume was larger and KPS scores were lower in the symptomatic than in the asymptomatic group [34].

The major weakness of the present study is that it was retrospective. As we discussed in our previous articles, the characteristics of patients receiving a particular treatment regimen are considered to have a major influence on treatment selection. This is an important issue when estimating the effects of treatments or exposures on outcomes using observational data. One approach to reducing or eliminating the effect of treatment selection bias and confounding effects is to use propensity score matching, which allows one to design and analyze an observational (non-randomized) study that mimics some of the characteristics of a randomized controlled trial. Therefore, in the present investigation, a case-matched study was also conducted by one of the authors (Y.S.), who did not participate in other aspects of this study and was blinded to final outcomes. Nevertheless, because this was a retrospective study, even with the application of case matching, biases in patient selection, original cancer treatments over time, follow-up (outcome, toxicity, and imaging), observers, and so on, could not be eliminated.

The other weakness of this study is the lack of information on tumor locations. It is absolutely crucial to describe tumor location when neurological symptoms are discussed. However, unfortunately, our retrospectively-accumulated database did not include information on tumor locations and, therefore, we could not examine the impact of this factor.

Moreover, there are several differences in welfare and health insurance systems between Japan and other countries, and these have major impacts on the cost of screening examinations such as MRI. Factoring in quality-adjusted life years for patients receiving versus not receiving routine scanning would be useful. 
A randomized controlled trial, in the near future, is necessary to test the hypothesis put forth herein.

\section{Conclusion}

Neurologically asymptomatic patients who underwent SRS for BM had better results than symptomatic patients in terms of maintenance of a good neurological state and a reduction in the neurological death rate. Our results indicate that screening for BM by CT/MRI may be beneficial for managing cancer patients. These observations merit further detailed research.

\section{Compliance with ethical standards}

Conflict of interest No actual or potential conflicts of interest exist.

Ethical standard Written informed consent was obtained from all patients.

Open Access This article is distributed under the terms of the Creative Commons Attribution 4.0 International License (http://creativecommons.org/licenses/by/4.0/), which permits unrestricted use, distribution, and reproduction in any medium, provided you give appropriate credit to the original author(s) and the source, provide a link to the Creative Commons license, and indicate if changes were made.

\section{References}

1. Ampil F, Calditto G, Milligan S, Mills G, Nanda A (2007) The elderly with synchronous non-small cell lung cancer and solitary brain metastasis: does palliative thoracic radiotherapy have a useful role? Lung Cancer 57:60-65

2. Gaspar L, Scott C, Rotman M et al (1997) Recursive partitioning analysis (RPA) of prognostic factors in three Radiation Therapy Oncology Group (RTOG) brain metastases trials. Int J Radiat Oncol Biol Phys 37:745-751

3. Mehta M, Tremont-Lukats IW (2002) Evaluation and management of brain metastasis. In: Proceedings of the 38th annual meeting of American Society of Clinical Oncology on 2002 educational book, pp 375-382

4. Penel N, Brichet A, Prevost B, Duhamel A, Assaker R, Dubois F et al (2001) Prognostic factors of synchronous brain metastases from lung cancer. Lung cancer 33:143-154

5. Hochstenbag MM, Twijnstra A, Hofman P et al (2003) MR-imaging of the brain of neurologic asymptomatic patients with large cell or adenocarcinoma of the lung. Does it influence prognosis and treatment? Lung cancer 42:189-193

6. Kim SY, Kim JS, Park HS et al (2005) Screening of brain metastasis with limited magnetic resonance imaging (MRI): clinical implications of using limited brain MRI during initial staging for non-small cell lung cancer patients. J Korean Med Sci 20:121-126

7. Figlin RA, Piantadosi S, Feld R (1988) Intracranial recurrence of carcinoma after complete surgical resection of stage I, II, and III non-small-cell lung cancer. N Engl J Med 318:1300-1305

8. Park HY, Kim YH, Kim H et al (2007) Routine screening by brain magnetic resonance imaging decreased the brain metastasis rate following surgery for lung adenocarcinoma. Lung cancer $58: 68-72$
9. Shi AA, Digumarthy SR, Temel JS et al (2006) Does initial staging or tumor histology better identify asymptomatic brain metastases in patients with non-small cell lung cancer? J Thorac Oncol $1: 205-210$

10. Yokoi K, Kamiya N, Matsuguma H et al (1999) Detection of brain metastasis in potentially operable non-small cell lung cancer: a comparison of CT and MRI. Chest 115:714-719

11. Davis PC, Hudgins PA, Peterman SB et al (1991) Diagnosis of cerebral metastases: double-dose delayed CT vs contrastenhanced MR imaging. Am J Neuroradiol 12:293-300

12. Lemiere C, Peretti-Viton P, Thomas $P$ et al (1995) Spreading evaluation in primitive bronchogenic carcinoma: benefit of cerebral MRI compared to CT scan. Eur J Cancer 31 A:1715

13. Sze G, Milano E, Johnson C et al (1990) Detection of brain metastases: comparison of contrast-enhanced MR with unenhanced MR and enhanced CT. Am J Neuroradiol 11:785-791

14. Yuh WT, Fisher DJ, Runge VM et al (1994) Phase III multicenter trial of high-dose gadoteridol in MR evaluation of brain metastases. Am J Neuroradiol 15:1037-1051

15. Cox JD, Yesner RA (1979) Adenocarcinoma of the lung: recent results from the Veterans Administration Lung Group. Am Rev Respir Dis 120:1025-1029

16. Newman SJ, Hansen HH (1974) Proceedings: frequency, diagnosis, and treatment of brain metastases in 247 consecutive patients with bronchogenic carcinoma. Cancer 33:492-496

17. Seute T, Leffers P, ten Velde GP et al (2004) Neurologic disorders in 432 consecutive patients with small cell lung carcinoma. Cancer 100:801-806

18. Sorensen JB, Hansen HH, Hansen M et al (1988) Brain metastases in adenocarcinoma of the lung: frequency, risk groups, and prognosis. J Clin Oncol 6:1474-1480

19. Vines EF, Le Pechoux C, Arriagada R (2003) Prophylactic cranial irradiation in small cell lung cancer. Semin Oncol 30:38-46

20. Lippitz B, Lindquist C, Paddick I et al (2014) Stereotactic radiosurgery in treatment of brain metastases: the current evidence. Cancer Treat Rev 40:48-86

21. McDermott MW, Sneed PK (2005) Radiosurgery in metastatic brain cancer. Neurosurgery 57(Suppl S4):45-53

22. Yamamoto M, Kawabe T, Higuchi Y et al (2012) Validity of three recently proposed prognostic grading indexes for breast cancer patients with radiosurgically treated brain metastases. Int J Radiat Oncol Biol Phys 84:1110-1115

23. Yamamoto M, Kawabe T, Sato Y et al (2013) A case-matched study of stereotactic radiosurgery for patients with multiple brain metastases: comparing treatment results for $1-4$ vs $\geq 5$ tumors: clinical article. J Neurosurg 118:1258-1268

24. Radiation Therapy Oncology Group (2015) Cooperative group common toxicity criteria. (https://www.rtog.org/ResearchAssociates/AdverseEventReporting/CooperativeGroupCommonToxicityCriteria.aspx). Accessed 1 Sep 2015

25. Gooley TA, Leisenring W, Crowley J et al (1999) Estimation of failure probabilities in the presence of competing risks: new representations of old estimators. Stat Med 18:695-706

26. Gray RJ (1988) A class of K-sample tests for comparing the cumulative incidence of a competing risk. Ann Stat 16:1141-1154

27. Satagopan JM, Ben-Porat L, Berwick M et al (2004) A note on competing risks in survival data analysis. $\mathrm{Br} \mathrm{J}$ Cancer 91:1229-1235

28. Fine JP, Gray RJ (1999) A: proportional hazards model for the subdistribution of a competing risk. J Am Stat Assoc 94:496-509

29. Bakoyannis G, Touloumi G (2012) Practical methods for competing risks data: a review. Stat Methods Med Res 21:257-272

30. Parsons LS (2015) Reducing bias in a propensity score matchedpair sample using greedy matching techniques. SAS. http:// www2.sas.com/proceedings/sugi26/p214-26.pdf. (Accessed 1 Sep 2015) 
31. D'Agostino RB Jr (1998) Propensity score methods for bias reduction in the comparison of a treatment to a non-randomized control group. Stat Med 17:2265-2281

32. Rosenbaum PR, Rubin DB (1983) The central role of the propensity score in observational studies for causal effects. Biometrika 70:41-55

33. Demange L, Tack L, Morel M et al (1989) Single brain metastasis of non-small cell lung carcinoma. Study of survival among 54 patients. Brit J Neurosurg 3:81-87

34. Sanchez de Cos J, Sojo Gonzalez MA, Montero MV et al (2009) Non-small cell lung cancer and silent brain metastasis. Survival and prognostic factors. Lung cancer 63:140-145

35. Yamamoto M, Serizawa T, Shuto T et al (2014) Stereotactic radiosurgery for patients with multiple brain metastases (JLGK0901): a multi-institutional prospective observational study. Lancet Oncol 15:387-395

36. Yokoi K, Miyazawa N, Arai T (1996) Brain metastasis in resected lung cancer: value of intensive follow-up with computed tomography. Ann Thorac Surg 61:546-550 (discussion 551)
37. Kuba S, Ishida M, Nakamura $Y$ et al (2014) Treatment and prognosis of breast cancer patients with brain metastases according to intrinsic subtype. Jpn J Clin Oncol 44:1025-1031

38. Colice GL, Birkmeyer JD, Black WC et al (1995) Cost-effectiveness of head CT in patients with lung cancer without clinical evidence of metastases. Chest 108:1264-1271

39. Cell lung Cancer (1997) The American Thoracic Society and The European Respiratory Society. Am J Respir Crit Care Med 156:320-332 - .

40. Barnholtz-Sloan JS, Sloan AE, Davis FG et al (2004) Incidence proportions of brain metastases in patients diagnosed (1973 to 2001) in the Metropolitan Detroit Cancer Surveillance System. J Clin Oncol 22:2865-2872

41. Shouten LJ, Rutten J, Huveneers HA et al (2002) Incidence of brain metastases in a cohort of patients with carcinoma of the breast, colon, kidney, and lung and melanoma. Cancer 94:2698-2705 